\title{
SCIENTIFIC PLANNING AND COMMANDING \\ OF THE ROSETTA PAYLOAD
}

\author{
D. KOSCHNY ${ }^{1, *}$, V. DHIRI ${ }^{1}$, K. WIRTH ${ }^{1}$, J. ZENDER ${ }^{1}$, R. SOLAZ ${ }^{1}$, R. HOOFS ${ }^{1}$, \\ R. LAUREIJS ${ }^{1}$, T.-M. HO ${ }^{1}$, B. DAVIDSSON ${ }^{2}$ and G. SCHWEHM ${ }^{1}$ \\ ${ }^{1}$ SCI-SO, ESA/ESTEC, Keplerlaan 1, NL-2200 Noordwijk, The Netherlands \\ ${ }^{2}$ Department of Astronomy and Space Physics, Box 515, S-75120 Uppsala, Sweden \\ (*Author for correspondence: E-mail: Detlef.Koschny@esa.int)
}

(Received 1 June 2006; Accepted in final form 1 December 2006)

\begin{abstract}
ESA's Rosetta mission was launched in March 2004 and is on its way to comet 67P/Churyumov-Gerasimenko, where it is scheduled to arrive in summer 2014. It comprises a payload of 12 scientific instruments and a Lander. All instruments are provided by Principal Investigators, which are responsible for their operations.

As for most ESA science missions, the ground segment of the mission consists of a Mission Operations Centre (MOC) and a Science Operations Centre (SOC). While the MOC is responsible for all spacecraft-related aspects and the final uplink of all command timelines to the spacecraft, the scientific operations of the instruments and the collection of the data and ingestion into the Planetary Science Archive are coordinated by the SOC. This paper focuses on the tasks of the SOC and in particular on the methodology and constraints to convert the scientific goals of the Rosetta mission to operational timelines.
\end{abstract}

Keywords: Rosetta, comet mission, science operations, RSOC, payload

\section{Introduction}

This paper gives an overview over the currently envisaged way of performing and coordinating the scientific operations of the Rosetta payload and it's Lander. The emphasis of this paper is how the operations are linked to fulfilling the scientific objectives of the mission.

\section{Mission Overview}

An overview over the mission is given in more detail elsewhere in this issue (Glassmeier et al., this issue). In summary: The spacecraft, consisting of an orbiter with 12 scientific instruments and a Lander (called PHILAE) with its own suite of instruments was launched in March 2004. A near-Earth instrument commissioning was performed to show that the instruments are functioning properly. 
The spacecraft performs several swing-by maneuvers where the gravity of the planets Earth $(3 \times)$ and Mars $(1 \times)$ are used to change the course of the spacecraft. While science measurements are performed during the swing-bys, the priority there lies with spacecraft activities. Two asteroid flybys are foreseen, one on 5 September 2008 at asteroid 2867 Steins, one on 10 July 2010 at asteroid 21 Lutetia. The asteroids are (secondary) science targets.

Rosetta is scheduled to arrive at the target comet in spring 2014. After a period of a few months to characterize the comet environment and its surface, it will deliver the PHILAE Lander. The aim is to deliver the Lander at a distance comet - sun of about 3.0 AU, which will be the case in autumn 2014. After that, the nominal science will be performed as described later in this paper, starting on 16 Nov 2014 at a solar distance of $2.97 \mathrm{AU}$.

The comet is a comparatively small body with an estimated $2.5 \mathrm{~km}$ radius. In a typical orbit, its gravitational attraction is about the same order of magnitude as the solar and planetary perturbations and the solar radiation pressure. This means that the spacecraft will not fly on Kepler orbits. It will also be difficult to predict the precise position of the spacecraft for more than a few days to weeks in advance. Thus a concept based on e.g. a 'frozen orbit', as used in many other planetary missions, cannot be used. In particular the pointing timeline for the spacecraft will need to be schedulable in a very flexible way, e.g. based on events, and it must be possibly to change it quite late in the planning cycle.

Another obvious issue when preparing a planning concept for a comet is the fact that the comet will change as it gets closer to the sun: It will become more active and additional perturbing forces on the spacecraft due to volatiles emanating from the comet's nucleus will increase. Also, dust jets may become active on the nucleus which would need to be avoided by the spacecraft.

\section{Spacecraft, Payload Accommodation, and Resulting Constraints}

The Rosetta spacecraft is a rectangular box of about $2 \mathrm{~m} \times 2 \mathrm{~m} \times 2 \mathrm{~m}$ in size, see Figures 1 and 2. The high-gain antenna (HGA) with its approximately $2.2 \mathrm{~m}$ diameter is mounted on the $+x$ side. The $+z$ axis is pointing upwards in the sketch. This is where most instrument apertures are pointing. Mounted at the side, on the $+y$ and $-y$ side, are two solar panels which span over a complete $32 \mathrm{~m}$. The PHILAE Lander is mounted on the $-x$ side.

The HGA has two degrees of freedom, allowing it to point more or less independently of the spacecraft body itself. There are certain mechanical constraints, however, restricting the antenna pointing positions. Also, the current strategy is that during slews of the spacecraft the HGA is fixed and only repointed after the end of a slew.

All radiators of spacecraft subsystems are mounted on the $+y$ and $-y$ sides, where the solar arrays are attached. Thus, when the solar arrays are pointed to the 


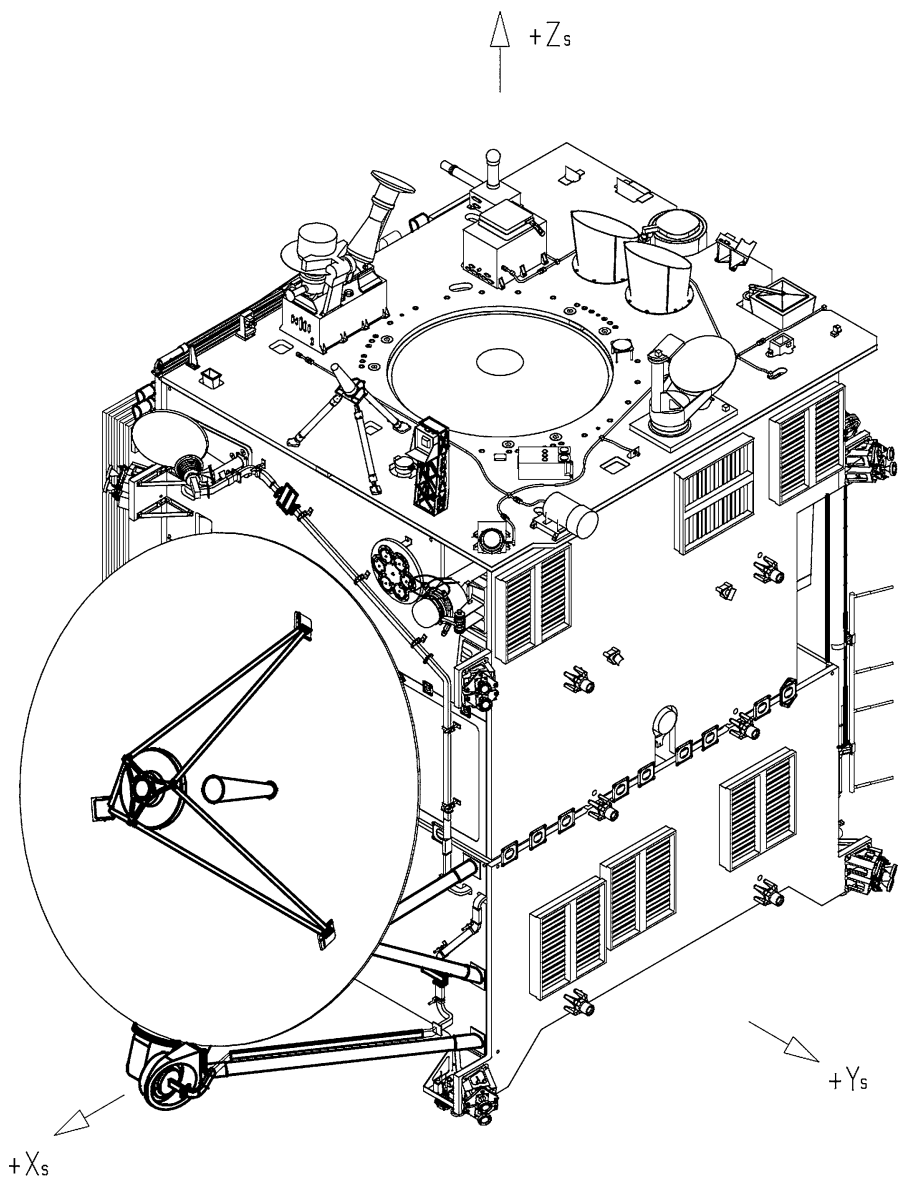

Figure 1. A sketch of the Rosetta spacecraft with the definition of the coordinate system. This shows the view on the $+x$ side, accommodating the high gain antenna. The solar arrays are shown folded to the spacecraft body.

sun, the $+/-y$ panels (sometimes called 'side panels') will not be illuminated and can cool the subsystems. During slews and maneuvers, however, slight illuminations of the side panels may occur.

The solar arrays have one degree of freedom, they can rotate $+270^{\circ}$ and $-90^{\circ}$ around the $y$-axis using the Solar Array Drive Mechanism (SADM). Thus it is in principle possible to point the $+z$ axis, which corresponds to the boresight of the instruments, to anywhere in the sky and keeping both the HGA pointed to the Earth (by using its two degrees of freedom) and the solar arrays perpendicular to the sun (by using the SADM and the $+z$ axis of the spacecraft). In reality, thermal constraints and mechanical constraints of the HGA limit the flexibility of the spacecraft additionally. 


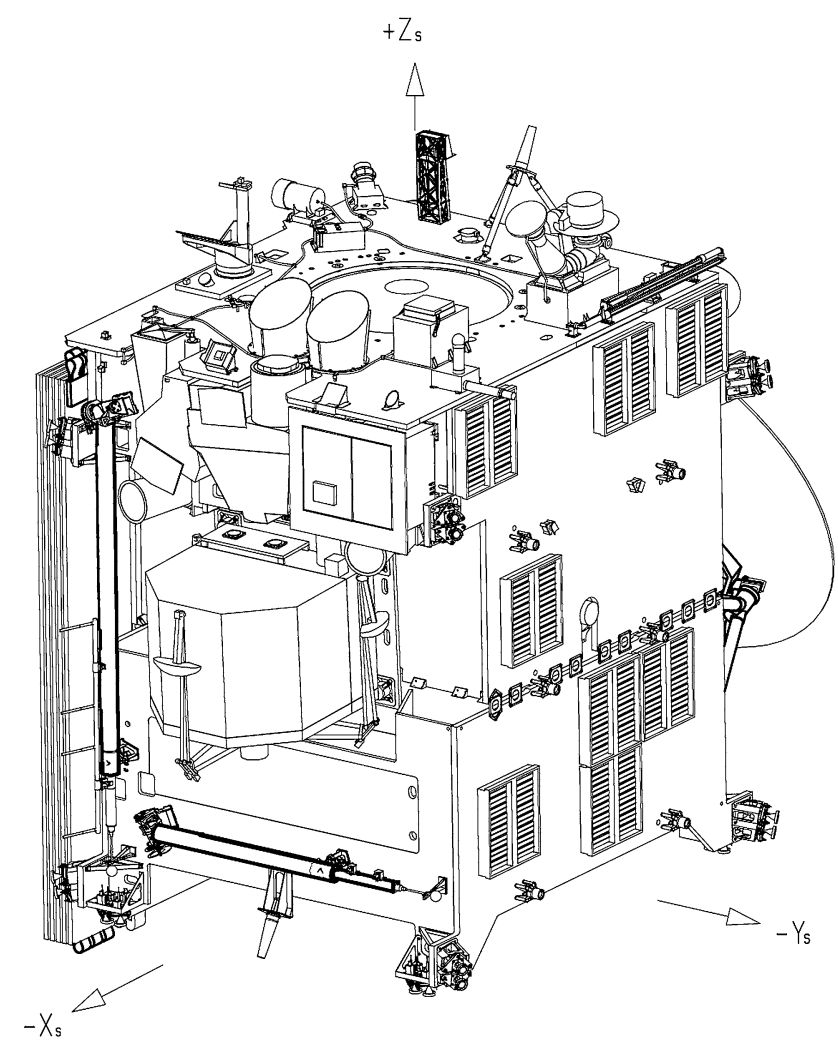

Figure 2. Sketch of Rosetta as seen from the $-x$ side. The box attached to the spacecraft is the Philae lander with its folded legs. The cylindrical and square baffles of the Navigation Cameras and the scientific camera OSIRIS point to the upward $(+z)$ direction.

Those instruments requiring the highest cooling are mounted on the $-z$ panel. They are the infrared spectrometer VIRTIS, and the scientific camera OSIRIS with its two camera systems NAC (Narrow Angle Camera) and WAC (Wide Angle Camera). Also, the radiators of the Navigation Camera (CAM) are pointing out the $-z$ panel. The PHILAE Lander is mounted on this side, as it also requires being in shadow for the complete mission before delivery onto the comet's surface. The spacecraft attitude will be such that this side is never illuminated, with possible short-time exceptions during the asteroid flybys.

Some of the thrusters are decoupled thermally from the spacecraft. In particular for the thrusters on the $-z$ panel this results in a thermal constraint to not point this side to the sun when closer than 1.8 Astronomical Units (AU) to the sun.

All remote sensing orbiter instruments are co-aligned and point towards the $+z$ axis, which is pointing to the top in the previous figures. To avoid the sun shining into the baffles of the instrument, the sun must not come too close to the $+z$ axis. The hard constraint comes from the UV spectrometer ALICE, stating that 
the sun must never be closer than $11^{\circ}$ from the $+z$ axis, which would damage the instrument. Other instruments have looser requirements. The OSIRIS NAC and WAC are designed such that they get straylight in the image when the sun is at $45^{\circ}$ to their optical axis. Also, the sun will shine into the blackened baffle, thus heating it up. The cameras have front doors, these must be closed if the sun shines into the baffles for too long a time to avoid thermal stress. The limiting angle for VIRTIS operations is $20^{\circ}$.

One of the major resource constraint is the communication from the spacecraft to the Earth. The High Gain Antenna of the spacecraft sends on X- and S-Band, the default receiving station is the ESA Deep-Space Antenna in New Norcia, Australia. The typical bitrate available for science is between 14 and $22 \mathrm{kBit}$ per second, with typical pass durations of 8 to 10 hours during the comet phase.

The spacecraft is designed such that starting at 3.2 AU to the sun, there is enough power to have all instruments operating in some typical mode. Closer than 1.4 AU to the sun, the total power has to be reduced to avoid overheating of the spacecraft. This, and the constrained downlink rate, leads to the need of detailed time-lining and the setting of priorities in instrument operations.

A summary of all spacecraft pointing constraints of the spacecraft is shown in Figure 3.

\section{The Ground Segment}

The complete ground segment of Rosetta consists of three main parties (see Figure 4): The experimenter teams (PI teams or instrument teams), the Rosetta Science Operations Centre (RSOC), and the Rosetta Missions Operations Centre (RMOC). The main tasks of each of these teams are:

- PI teams: support the long-term scientific planning of the mission, supported by the RSOC prepare the detailed operational timelines for their instrument, ensure that the instrument-specific constraints are not violated, perform any software maintance of their instruments, analyze the returned data from their instruments, produce scientific results based on those data, prepare the data for ingestion into the long-term ESA archive (the Planetary Science Archive, PSA).

- RSOC: Coordinate the long-term scientific planning of the mission, support the PI teams in the preparation of the detailed operational timelines, consolidate the operational timelines from the complete payload and perform resource checking, deliver this timeline to the RMOC. Support the PI teams in the definition of the long-term archive products, ingest the data into the Planetary Science Archive.

- RMOC: Responsible for all spacecraft-related issues. Provides flight dynamics services, i.e. preparation and commanding of the spacecraft attitude 


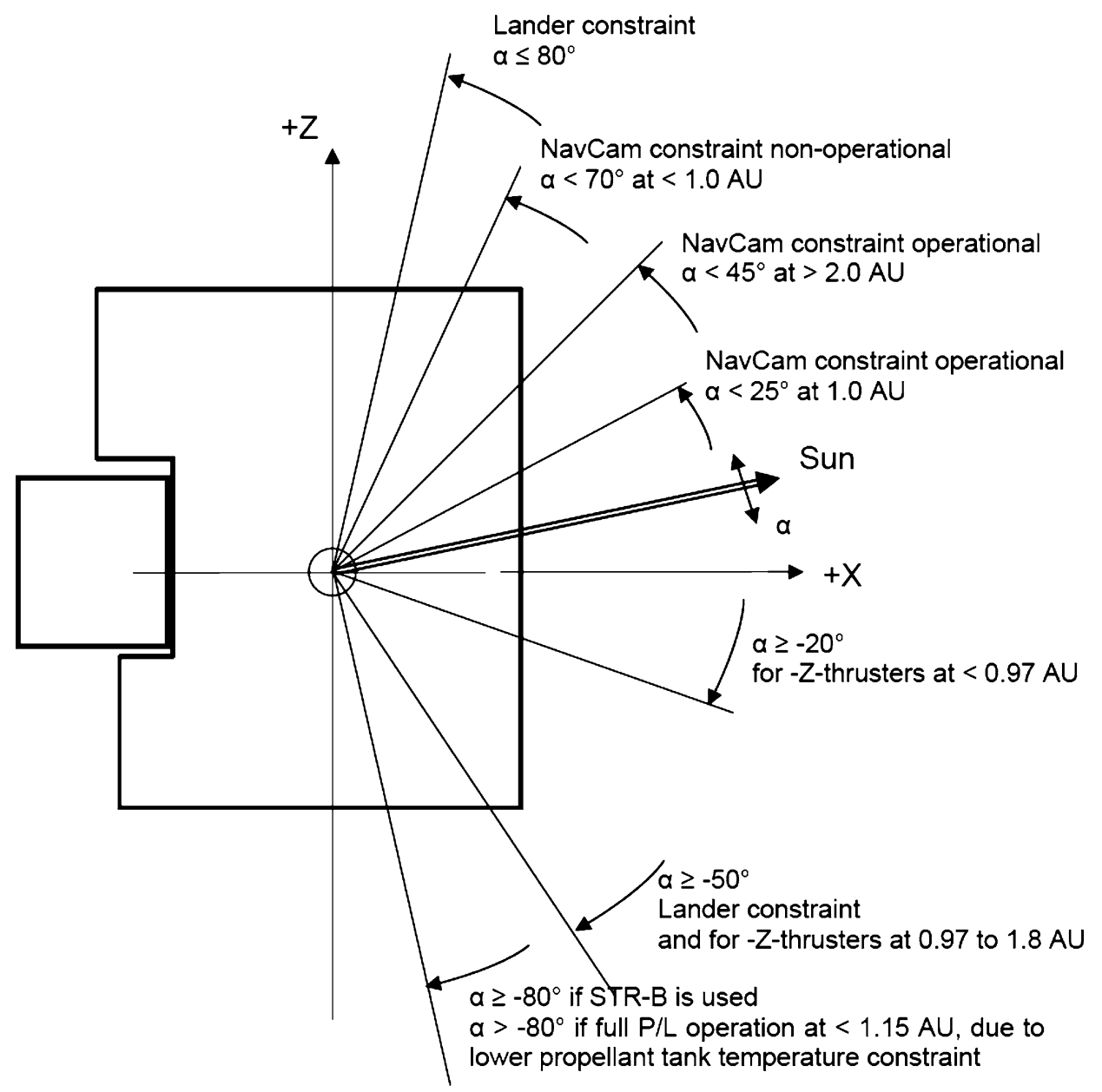

Figure 3. Summary of the top-level pointing constraints of the spacecraft. The box is a sketch of the spacecraft body, with the PHILAE Lander attached on the left $(-x)$ side. In general, the sun can only come from the right side to keep the $-x$ panel cool.

and trajectory, wheel off-loadings, communications with the spacecraft. The RMOC consolidates the payload timeline as provided by the RSOC with all commands needed to control the spacecraft itself. RMOC also ensures the proper downlink of all data and provides it to the teams via the so-called Data Disposition System (DDS).

The operational interfaces are implemented via computer-readable files, which are transferred via secure means. The planning for the mission at the comet is done in three cycles which are outlined in a later section. 


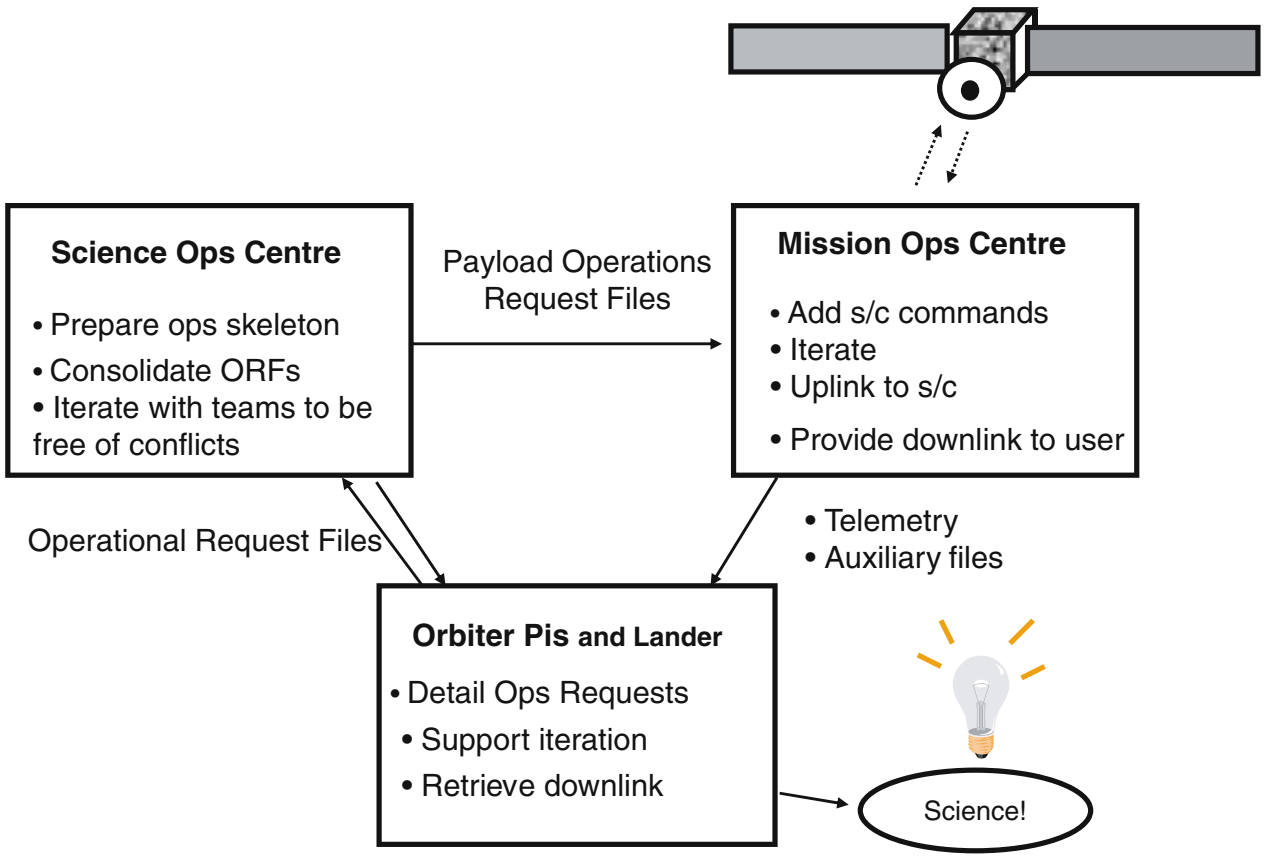

Figure 4. A block diagram of the Rosetta ground segment, showing the different participants in the process. For a more detailed explanation, see text.

\section{Structuring the Mission}

The Rosetta mission is divided into so-called mission phases. Each mission phase has a defined start and end date or event. Examples for mission phases are commissioning, Earth swing-by, etc. A complete table of the mission phases can be found in Glassmeier et al. (this issue).

Within a mission phase, different mission scenarios are possible. A mission scenario is a time in the order of days to weeks, linked to a given set of science goals. Examples for mission scenarios are "Close encounter (CLOSE)" or "Gas/dust jet sampling (JET)". For "Gas/dust jet sampling" the corresponding science goal would obviously be "Sample gas and dust in a jet and analyse it". For each mission scenario, a certain number of experiments would be on in a certain operating mode. Mission scenarios can be performed several times per phase, e.g. in the "Comet low activity phase (LOW)" there could be several scenarios called "Close encounter". Each scenario can have a "priority experiment" which can drive the trajectory and pointing requests. This does not mean, however, that other experiments are not on. All it means is that this experiment (or set of experiments, e.g. remote sensing) are planned first. The description of the experiment operations in a mission scenario together with the associated s/c trajectory and pointing information is called Master Science Plan (MSP). 
The mission scenarios per phase are proposed by the Rosetta Science Operations Centre and discussed and confirmed by the Science Working Team (SWT), which is composed of all Principle Investigators of the experiments. The mission scenarios can be seen like "puzzle pieces" or modules that can be prepared in advance and used when required.

The RMOC expects before each mission phase a so-called Science Activity Plan (SAP) for this phase. The SAP is a time-ordered listing of mission scenarios, describing on top level what should be done when, The SAP will be produced by the RSOC together with the SWT as part of the long-term planning cycle.

\section{Planning During the Cruise Phase}

A number of scientific observations will be performed already during the asteroid flybys and planetary swingbys. These are planned as 'one-off' activities following a schedule of typically 6 to 12 months duration, where activities are iterated between the PI teams, the Rosetta Science Operations Centre, and the Rosetta Mission Operations Centre. A more detailed description of these operations is given in Wirth et al. (2006). This paper will in the following focus on the detailed planning for the comet phase.

\section{Planning During the Comet Phase}

\section{INTRODUCTION}

The comet observation phases will be planned via three cycles, long-term, mediumterm and short-term planning. The long-term planning cycle covers all long-term planning aspects, in particular the development of mission scenarios and the development of the Science Activity Plan, i.e. a time-ordered listing of mission scenarios, for each mission phase. To link the scientific planning to the operational constraints, the so-called 'Science Themes concept' will be used. This is detailed in a dedicated section later in this paper. The top-level science and technology goals are split down into so-called Science Themes and Sub-Themes, which in turn are split up into Detailed Science Objectives. These are linked to real measurements, resulting in different sets of observations.

A science themes spreadsheet will be provided to the experiment teams, which are requested to fill in the parts relevant to their experiments and discuss it with the other teams. This will be done via regular telecons, starting long before the actual arrival at the comet. The end result will be a detailed mapping between the science goals and the possible observations.

There will be a so-called training period starting about 2 years before comet encounter. During this period, the complete planning process down to the lowest 
level (the POR files) is performed using a model comet in order to exercise the planning process and to develop an operations baseline.

When arriving at the comet, the medium-term planning and the short-term planning will occur. The medium-term planning will fix the resources and last roughly one month, the short-term planning allows instantiating parameters in the operational requests as produced during the medium-term planning and will be roughly on a weekly basis. Other than on previous missions, the precise beginning and end time of the planning cycles will not be fixed relative to the calendar. Rather, it will be driven by operational activities, i.e. a short-term plan will not end in the middle of an operational scenario but either before or after it.

The following text first gives an overview over goals, activities and schedule of the planning cycles in bullet form. After that, one dedicated section per planning level describes the activities in more detail.

\section{Long-term planning:}

- Goal: A baseline Science Activity Plan (SAP) and scenario descriptions shall be available before each mission phase.

- Activities:

- Scenario development (scenario description, i.e. Master Science Plan (MSP))

- SAP development

- Schedule: Has started already. Covers the complete mission.

- Output:

- Mission scenario documents, containing a scenario description, i.e. Master Science Plan (MSP), for each scenario, with pointing and trajectory information and operations at least down to mode level

- SAP (a time-ordered listing of the mission scenarios)

Training phase:

- Goal: Ensure that the planning process was done at least once in all detail. Exercise the planning process and develop an operations baseline.

- Activities:

- For each mission scenario, generate baseline Payload Operational Request (POR) file (based on baseline MSP, baseline scenarios).

- Build up a "library" for operations.

- Schedule: Ca. 2 years before the Comet Acquisition Point is reached. Covers the comet phases.

- Output:

- Detailed planning (POR file, pointing, trajectory) for each mission scenario, based on dummy comet. 
Medium and short-term planning:

- Goal: Actually perform the science operations at the comet.

- Activities:

- Generate requests to flight dynamics (Scenario Paramter Lists - SPLs, and Pointing Request Files - PTRs)

- POR file generation

- Update SAP and MSPs only if needed.

- Schedule: After Comet Acquisition Point is reached, until end of mission. Typically one medium-term planning input is provided per month, one shortterm planning period will cover one week.

- Output:

- Detailed planning (POR file, pointing, trajectory) for each scenario, based on the real comet.

\section{LONG-TERM PLANNING}

The goal of the long-term planning cycle is to construct a baseline Science Activity Plan (SAP) and scenario descriptions before each mission phase. To do this, two main activities have to be performed:

(a) Scenario development (scenario description, i.e. Master Science Plan)

(b) Science Activity Plan (SAP) development

A graphical representation of the tasks during the scenario development are given in Figure 5.

The long-term planning process starts with the formulation of the top-level science goals and their derived observation requirements. These have been documented in User Manuals by the experimenter teams. Additionally, top-level operational requests are communicated to the RSOC via e-mail, fax, or during SWT/SOWG meetings.

RSOC will group the science requests by science disciplines, e.g. remote sensing, or scientific phenomena, e.g. cometary outbursts. For example, there might be individual requests to fly through a cometary jet, from both gas and dust sampling experiments. It is obvious that these can be grouped and performed during one given trajectory around the comet. The RSOC will define a mission scenario for this, e.g. "gas/dust jet sampling". It will require a certain trajectory and pointing/slewing profile. Constraints, e.g. on the downloadable data volume, available power and interference between the different instruments, must be taken into account. This process of linking the operational (engineering) constraints and the science requirements is called the 'Science Themes concept' and described in more detail later in this paper in a dedicated section. 


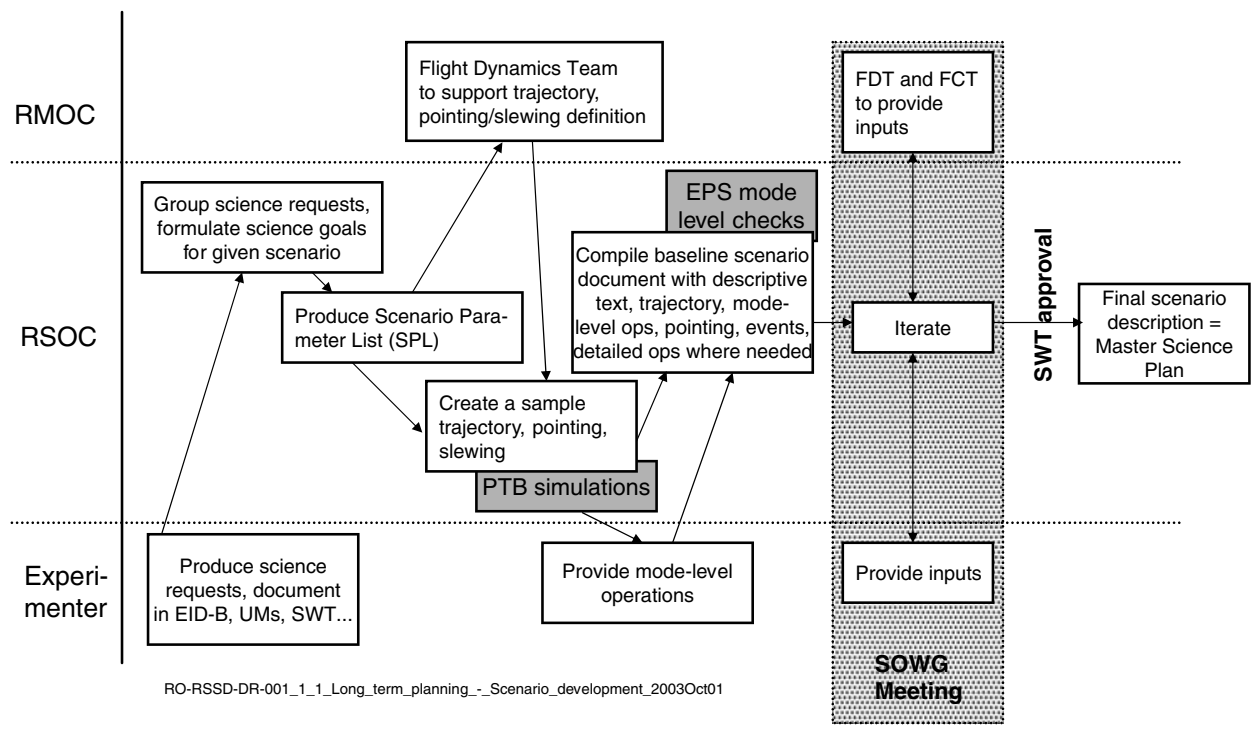

Figure 5. Graphical overview over the long-term planning cycle - Scenario development.

The RSOC will produce consolidated Scenario Parameter Lists (cSPLs) which, together with Pointing Request (PTR) files, define the interface between the RSOC and the Flight Dynamics Team (FDT). The cSPLs define items like illumination conditions, required distances to landmarks, pointing/slewing patterns, etc. The cSPLs are based on input from the PI teams, which are documented in so-called observation requests.

In addition, the RSOC will already generate draft mode-level experiment operations in order to check that the constraints are observed.

The RSOC will develop a trajectory and a pointing/slewing profile using its planning software (the Rosetta PTB $=$ Project Test Bed). The pointing requests will be written into a so-called Pointing Request (PTR) file which is computer readable. After agreement by the PI teams, these files will be sent to the FDT.

The FDT will check the cSPLs and support the development and comment on the trajectory and pointing/slewing profiles as documented in the PTR files and ensure that no major problems can occur.

The resulting profile will be filled out by the RSOC and the experimenter teams with at least mode-level operations of the experiments, while the constraints will be observed. The mode-level operations will be checked for potential conflicts using the Experiment Planning System (EPS).

The RSOC will compile from this information a baseline scenario document (Master Science Plan) with

- a descriptive text, 
- a trajectory request (included in the Scenario Parameter List) and a reference trajectory file,

- a pointing request file and a reference pointing file,

- a reference event file, or the baseline event file from FDT,

- at least mode-level operations of the experiments.

Each scenario will be iterated by RSOC with the experimenters and the RMOC. Typically this is done in a workshop organised during a Rosetta Science Operations Working Group (SOWG) meeting (e.g. the workshops on the "interference scenario" and the "pointing scenario" conducted during SOWG \#5 and SOWG \#6). The final MSP needs to be approved by the SWT.

The second part of the long-term planning process is performed in parallel, the Science Activity Plan development.

One of the inputs for the SAP development is some baseline information on the trajectories of the individual mission scenarios. The reason is that the spacecraft trajectory has to fit together in a reasonable way. For example, it would take a lot of fuel to go directly from an equatorial orbit into a polar orbit.

Also, the scenarios will be sorted somehow as a function of comet activity. Obviously, the sampling of a jet may not be possible in the beginning of the mission if no jets are active yet.

Thus, the activities during the SAP development can be summarised as follows:

From the top-level science inputs documented in the experimenter teams' documentation (EID-Bs, User Manuals, e-mails) and the preliminary information on trajectories, the RSOC will prioritise the scenarios. Those which impose the lowest risk on the spacecraft and/or experiments will be done in the beginning. In addition, the scenarios will be ordered according to its requirements concerning comet activity.

RSOC will produce a draft SAP, which will be iterated with both the RMOC and the experimenter teams, typically in working group meetings during a SOWG meeting. The final SAP needs to be approved by the SWT. The outcome will be one Science Activity Plan for each mission phase.

A graphical overview of the SAP development is given in Figure 6.

\section{Training Phase}

The goal of the training phase is to ensure that the complete planning has been exercised at least once. It will take place in the time span from about 3 years starting in January 2010 before comet acquisition until the short-term planning starts. Based on the scenarios and the SAP developed during the long-term planning process and using a sample comet, the planning for each scenario will be detailed down to the level of the Payload Operations Request (POR) files following the description in the next section on medium- and short-term planning. 


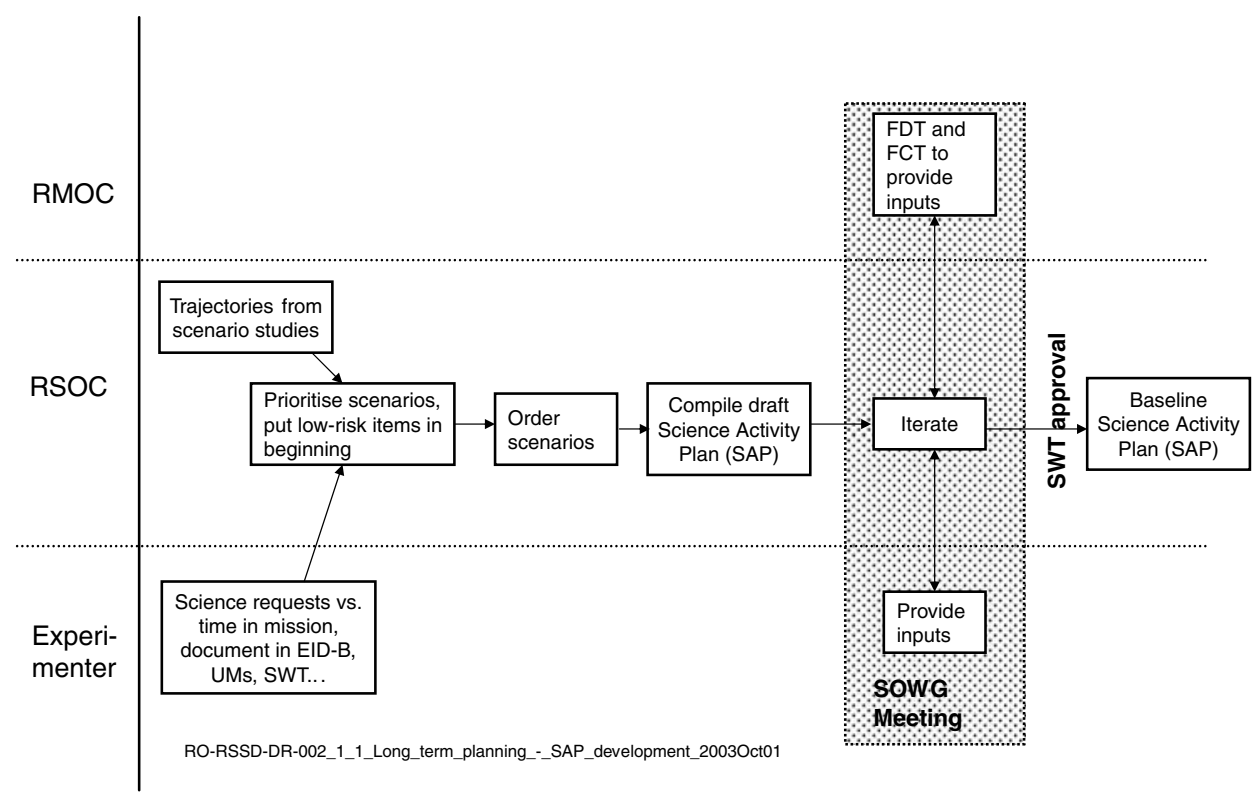

Figure 6. Graphical overview over the long-term planning cycle-SAP development.

The result will be a library of planning modules that can be quickly adapted to the real comet once its properties have been measured. For a graphical overview see Figure $\mathrm{xx}$ in the next section.

\section{Medium And Short-Term Planning}

The medium and short-term planning cycle will start as soon as the real comet is known, i.e. after the Comet Acquisition Point was reached. The medium-term planning comprises the generation of input for Flight Dynamics and its iteration. This will happen on a roughly monthly basis, with the detailed start and end times defined by the beginning/end of a mission scenario.

The RSOC will start from the scenarios as defined during the long-term planning cycle. The first step will be to confirm or update the cSPLs and PTRs to take into account new knowledge about the requirements from the experimenter teams. These will be given to the FDT, who will produce new predicted trajectory files $(x / y / z$ position), pointing/slewing profiles (quaternions) and event files (EVF files).

Using this information, RSOC will go through the scenario documents (Master Science Plans) and propose updates and changes in the mode-level operations to the experimenter teams. These will update the mode-level operations and fill in detailed operations down to the level of telecommand sequences. If required, they will also update or add trajectory and pointing requests. 
The RSOC takes this information and compiles it to generate a consolidated POR file and final trajectory and pointing/slewing requests (PTR files). Using the EPS software, RSOC will check for conflicts and, if required, iterate with the experimenter teams. The final cSPL/PTR will be given to the Flight Dynamics Team. The FDT will generate a final trajectory and pointing/slewing profile. RSOC will iterate the operations of the experiments, based on this trajectory and pointing/slewing profile, with the experimenter teams and the FCT. The final outcome will be one or several POR files for each scenario. After the medium-term planning cycles, the resource requirements to the spacecraft will be fixed, resources being pointing, thermal constraints, power, data volume/rate, number of telecommands per planning cycle.

The short-term planning comprises mainly the instantiation of parameters in the POR files. It will happen on a time scale of several days to one week, with the precise start/end times defined by the beginning/end of a mission scenario. The process itself is similar to that performed during the Training phase. However, it is expected that less time for iterations will be available. Also, the measured comet parameters will be used. A graphical overview is given in Figure 7. The dashed boxes indicate that it is expected that only limited time will be available to perform these tasks.

Depending on the physical parameters and activity profile of the comet, the scenarios and possibly even the Science Activity Plan have to be redefined. If this is the case, the activities as shown in Figures 5 and 6 will be performed again.

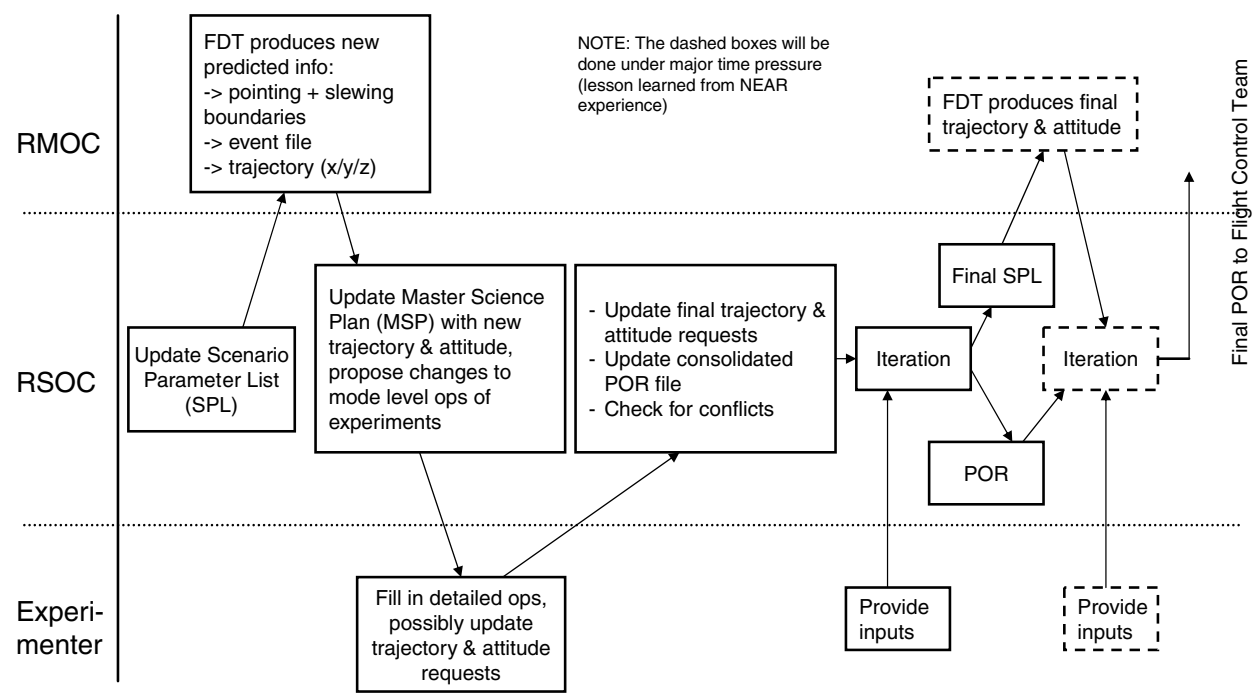

RO-RSSD-DR-004_1_1_Short_term_planning_-_POR_file_generation_20030ct01

Figure 7. Graphical overview over the medium and short-term planning cycle - POR file generation. 
The short-term planning process will be organised in so-called "planning cycles". These will have consecutive numbers. Each one of them will have deadlines for the different interfaces defined. These are indicated in Figure 8, where the above block diagram is depicted as a bar chart. In this example, it is assumed that the uplink of the Mission Timeline is done on a Wednesday morning. It is also assumed that the covered time span of this planning will be one week, i.e. uplinking is performed every week. Note that due to the limited capacity of the MTL it is expected that more than one upload will be necessary to cover the one week. Also, as mentioned before, the real starting/end date of a short-term cycle will depend on the operational scenario.

Calculating backwards, the first deadline, when the experimenter teams have to send their Operational Request File (called OIOR $=$ Orbiter Instrument Operational Request or LOR $=$ Lander Operational Request) to the RSOC, would be on the Friday morning, nearly two weeks earlier. The receipt of these files will be acknowledged by the ORF-A software, within a few minutes. The RSOC requires one and only one file (i.e. one OIOR) from each experimenter team, covering the complete duration of the planning cycle. It will then perform the planning as depicted in Figure 7, including iterations with the experimenter teams, Flight Dynamics and the Flight Control Team.

Obviously, several of these planning cycles will be going on in parallel.

The acceptance of an OIOR or LOR file from the experimenter teams for the planning is implicitly given when the final POR file is put on the server.

\section{Contingency Handling}

Procedures for contingency handling within an experiment must be provided by the experiment teams. In case that telemetry indicates a problem with an experiment, the RMOC will contact the respective PI and the RSOC to find a solution. The RSOC will archive the relevant information.

If measurements cannot be performed due to problems with the $\mathrm{s} / \mathrm{c}$ or an experiment, the main priority will be to continue with the next scenarios. It will not be priority to recover the measurements. An example: Assume that a "close encounter scenario" was planned for 5-12 March, to investigate in detail the surface profile of an active area using the remote sensing experiments. On 13 March, a "gas/dust jet sampling scenario" was supposed to start. If on 10 March problems with the measurements of one of the remote sensing experiments occur and cannot be recovered until 13 March, the priority will be given to the start of the "gas/dust jet sampling scenario". It will not be attempted to repeat the measurements of the close encounter scenario. The reason is that the scenarios are closely linked to the $\mathrm{s} / \mathrm{c}$ trajectory. Repeating a scenario or trying to recover lost measurements would mean a complete re-planning of the future trajectory. This will be avoided whenever possible. 
Rosetta Short-Term Planning: Sample Schedule

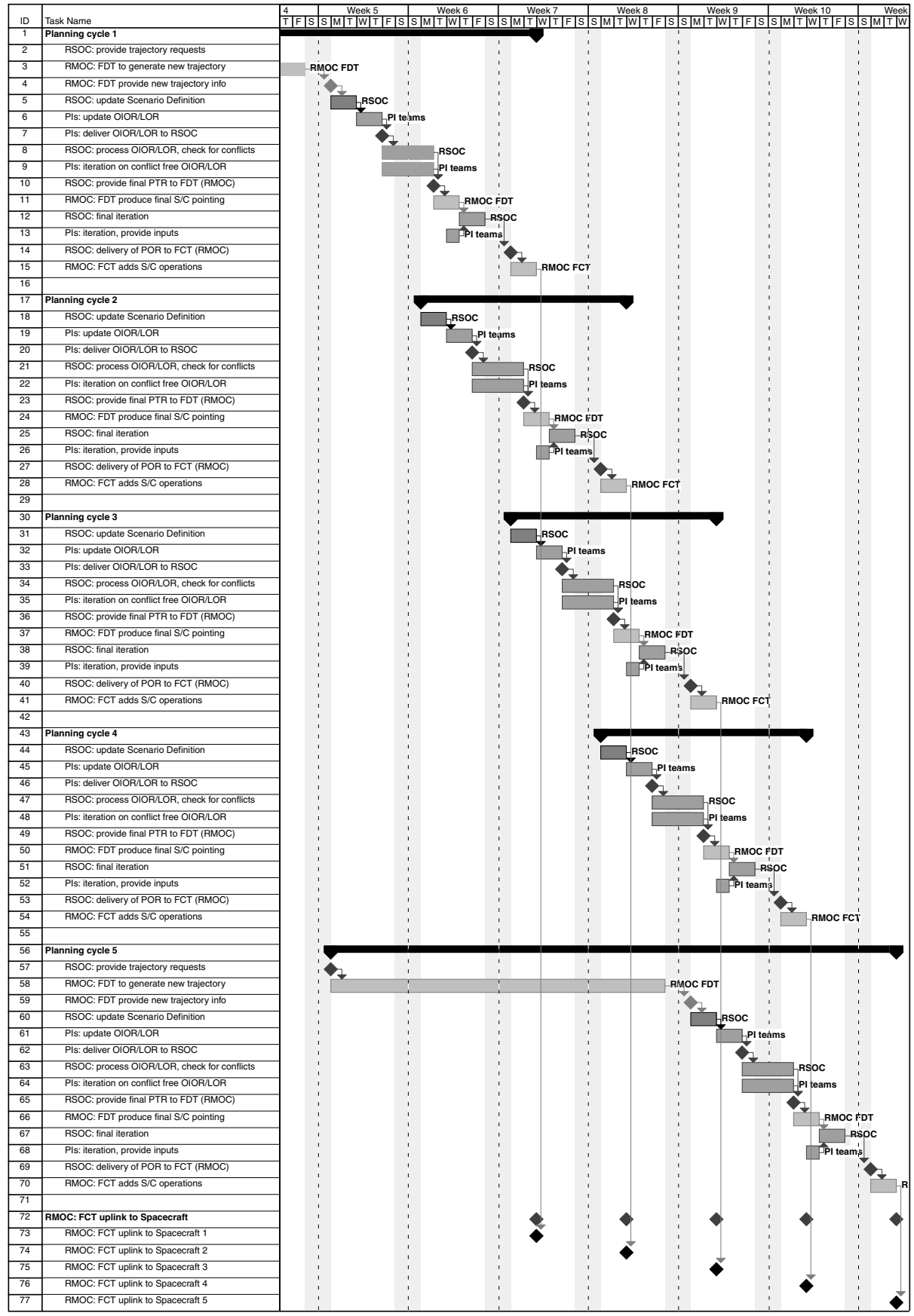

Figure 8. Detailed schedule of 5 planning cycles during the nominal short-term planning process. Note that the actual starting and end dates for a short-term planning cycle will be adapted to the operational mission scenario. 


\section{CONFLict Solving}

Conflicts will be found in the planning process when the RSOC analyses the consolidated planning files (OIOR/LOR files, PTR files). Conflicts are generated whenever certain constraints are violated. These constraints are defined in the so-called Experiment Description Files (EDFs), which act as a database to model the experiments. For example: In the EDF of the atomic force microscope MIDAS, there might be a constraint describing that MIDAS should not be in Scan mode whenever the cooler of the spectrometer VIRTIS is on. The EPS will check for this constraint. If it finds out that it is violated it will write a corresponding message into the conflict (CONF) file.

If a conflict is flagged by the planning software, RSOC will propose changes to the input timelines of the concerned teams and iterate a conflict-free timeline with them. After the iteration, the experimenter teams will provide an updated OIOR/LOR file to the RSOC. If no solution is found during this iteration, the issue will be discussed with the SWT, provided that there is enough time. The final decision authority (also to reject a timeline) is with the Project Scientist.

To avoid conflicts to appear based on invalid constraints, the EDFs used by the RSOC for the planning process will be approved by the Principle Investigator or Lander Lead Scientist. The EDFs will be under configuration control.

\section{The Science Themes Concept}

\section{General Overview}

The science operations planning are structured according to Figure 9. There are two sides to the planning: The science side with their science goals, and the operations side with the constraints of the spacecraft and the environment. Both of these are grouped and detailed, until one arrives at 'observations'. Observations are building blocks, which perform certain measurements contributing to a detailed science goal. It is possible that more than one observation or a repeat of observations over more than one orbit is needed to completely fulfill a science goal.

Also, it is possible that observations contribute to more than one science goal.

From the operational side, there will be operational constraints coming from the spacecraft or other experiments. Also, the trajectory and other environmental conditions like the illumination will influence the timing of operations. These will influence the "mission scenarios" that contain of a group of observations.

This abstract structure will be filled up with real science objectives, observations, and scenarios during the long-term planning process.

It is emphasized that the definition of the science themes and their breakdown to detailed observations is the task of the PI teams. RSOC only coordinates this process and provides methods and tools to fill in and later track the science themes. 


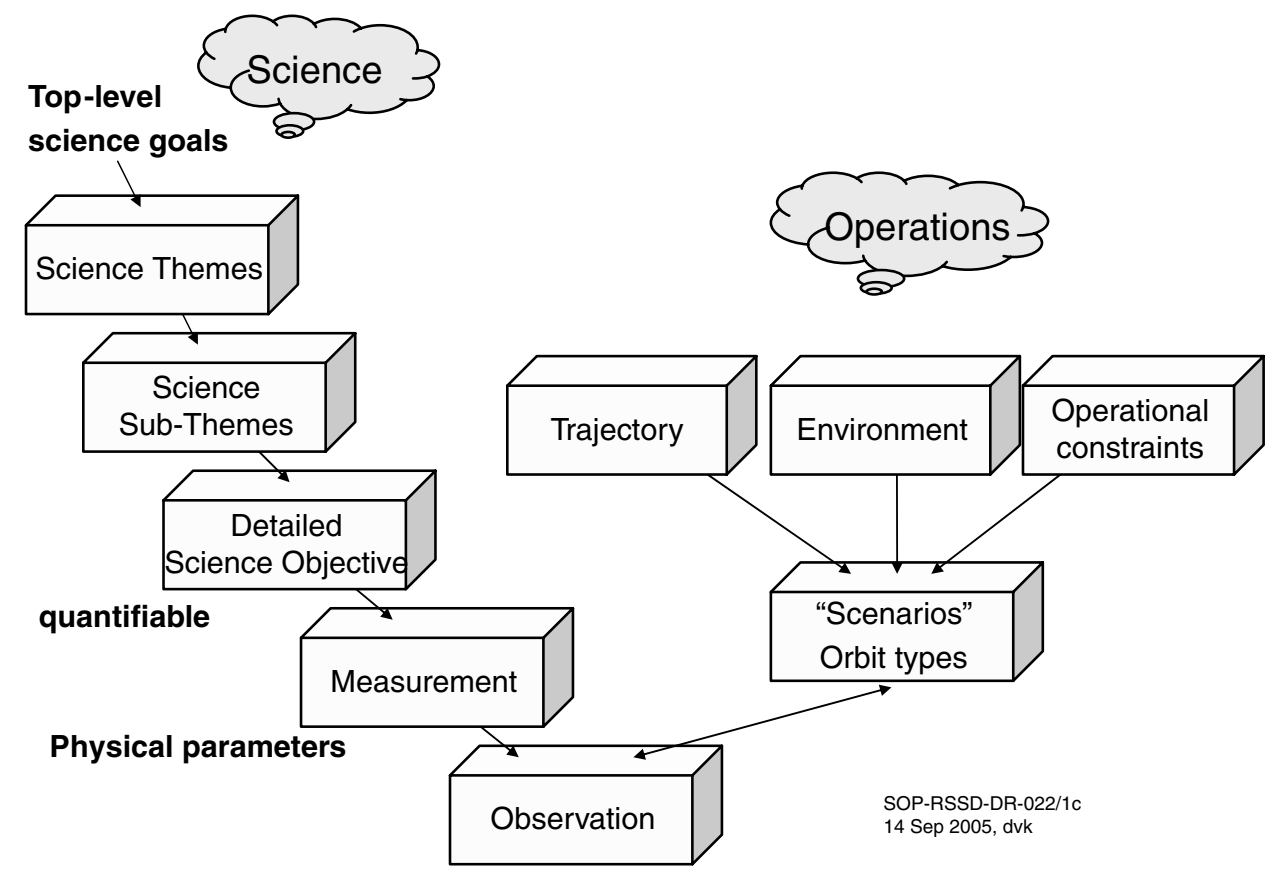

Figure 9. The Science Themes concept. For a detailed explanation, see the text.

\section{The Science Side \pm Science Themes And Sub-themes}

For the large scientific planetary missions, so-called top-level mission science goals are available. These are grouped and sorted to make sense from a scientific point of view. Typically, these are defined in discussions between the SOC and the Interdisciplinary Scientists. For example, for VEX the Science Themes are 'Atmospheric Dynamics', 'Atmospheric Structure', 'Atmospheric Composition and Chemistry', 'Surface Properties and Surface-Atmosphere Interaction', 'Plasma Environment and Escape Processes'. Obviously, for VEX many Science Themes are related to the atmosphere. To give an example for the Sub-Themes, the Theme 'Surface Properties...' was subdivided into 'Geology', 'Surface-Atmosphere Interaction', 'Seismic Activity', 'Volcanology'.

The top-level science themes have been derived for Rosetta in 2003/2004, with the help of the Interdisciplinary Scientists. They are derived by the initially given science goals of the mission, with the difference that the themes are all on the same level of importance. The detailed scientific objectives of each of the instruments will need to be mapped by the instrument teams, and from that detailed scientific objectives will have to be derived. This will be done by teleconferences with the experiment teams and in Science Operations Working Group meetings.

The currently defined Science Themes and Sub-Themes for Rosetta can be found in Table I. 
TABLE I

The rosetta science themes and sub-themes.

\begin{tabular}{|c|c|c|c|}
\hline & Science theme & & Sub-theme \\
\hline \multirow[t]{5}{*}{1} & Global characterization of the nucleus & A & Nucleus dynamics \\
\hline & & $\mathrm{B}$ & $\begin{array}{l}\text { Nucleus mass, gravity, shape, volume, and } \\
\text { bulk density }\end{array}$ \\
\hline & & $\mathrm{C}$ & Nucleus topography and morphology \\
\hline & & $\mathrm{D}$ & $\begin{array}{l}\text { Near-surface distribution of minerals, } \\
\text { organics, and ices }\end{array}$ \\
\hline & & $\mathrm{E}$ & Internal nucleus structure \\
\hline \multirow[t]{3}{*}{2} & Composition of the nucleus material & A & Composition of near-surface minerals \\
\hline & & $\mathrm{B}$ & Composition of near-surface organics \\
\hline & & $\mathrm{C}$ & Composition of near-surface ices \\
\hline \multirow[t]{5}{*}{3} & Physical properties of nucleus material & A & $\begin{array}{l}\text { Microphysical properties of nuclear } \\
\text { material }\end{array}$ \\
\hline & & $\mathrm{B}$ & $\begin{array}{l}\text { Electric and magnetic properties of nucleus } \\
\text { material }\end{array}$ \\
\hline & & $\mathrm{C}$ & Thermal properties of the nucleus material \\
\hline & & $\mathrm{D}$ & $\begin{array}{l}\text { Mechanical properties of the nucleus } \\
\text { material }\end{array}$ \\
\hline & & $\mathrm{E}$ & Optical properties of the nucleus material \\
\hline \multirow[t]{3}{*}{4} & Development of cometary activity and & A & Properties of regions emitting gas and dust \\
\hline & & $\mathrm{B}$ & $\begin{array}{l}\text { Temporal evolution of the surface and } \\
\text { inner coma }\end{array}$ \\
\hline & & $\mathrm{C}$ & Properties of structures in the inner coma \\
\hline \multirow[t]{4}{*}{5} & Composition of coma and tail material & A & Composition of neutral gaseous species \\
\hline & & $\mathrm{B}$ & Composition of ions \\
\hline & & $\mathrm{C}$ & Composition of solid particles \\
\hline & & $\mathrm{D}$ & $\begin{array}{l}\text { Chemical reactions between neutrals, ions, } \\
\text { and grain surfaces }\end{array}$ \\
\hline \multirow[t]{3}{*}{6} & Physical properties of coma and tail & A & Physical properties of neutral gas \\
\hline & & $\mathrm{B}$ & Physical properties of ions and electrons \\
\hline & & $\mathrm{C}$ & Physical properties of solid particles \\
\hline \multirow[t]{2}{*}{7} & Interaction of cometary material with & A & Properties of the plasma tail \\
\hline & & $\mathrm{B}$ & Properties of the ionosphere \\
\hline
\end{tabular}


TABLE 1

(Continued)

\begin{tabular}{llll}
\hline \multicolumn{2}{c}{ Science theme } & & Sub-theme \\
\hline 8 Asteroids & A & Dynamical and rotational properties \\
& B & Global characteristics \\
& C & Surface properties \\
& D & Interactions with the solar wind \\
& E & The asteroid environment \\
& A & Science during earth swingbys \\
& & \\
Science not directly related to comets & B & \\
& C & Science during mars swingby \\
& D & Science during cruise phase \\
& D & Science during circum-nucleus orbit phase \\
\hline
\end{tabular}

\section{Detailed Science ObJectives}

From the Science Sub-Themes, detailed Science Objectives will be derived. The idea there is that these are quantifiable, i.e. it should be possible to put numbers on them. A typical Science Objective could be "Map the PHILAE landing site from at least two different illumination angles in the visible and the infrared with a resolution of at least $10 \mathrm{~cm}$. The elevation of the Sun should be between 30 and 60 deg'. These detailed Science Objectives have to be defined by the PI teams before the start of the detailed mission planning.

\section{MEASUREMEnTs AND OBSERVATIONS}

A Measurement is whatever it takes to actually fulfill a detailed Science Objective. For example, for the example given above, 'Map the PHILAE landing site...' this could be 'Obtain a mosaic of the site in two different orbits, using all filters a, b, $c$, of OSIRIS, from a distance of less than $t b c$ kilometers. While doing this, obtain spectra with VIRTIS'. The Observation is the smallest possible operational item that can be done consecutively. In this case more than one Observation would be needed to do the measurement: 'In orbit xx, start acquiring images when $2 \mathrm{~km}$ away from the site until $2 \mathrm{~km}$ after closest distance. Have VIRTIS operating in mode xx' - 'In orbit $\mathrm{xx}+1$, start acquiring images... etc.'. Note that all these examples are for illustration only. The real Rosetta Observations must still be discussed after the detailed Science Objectives are defined. This is a process ongoing during the time of writing of this paper.

Observations can be described with a generic Scenario Parameter List (SPL) describing the pointing and an associated generic operational request file describing 
the payload operations. Several observations that occur consecutively can be grouped per orbit.

\section{The Operations Side \pm Trajectory, EnVironment,}

\section{OPERATIONAL CONSTRAINTS}

On the operational side, there are a number of constraints that need to be considered in the planning. The trajectory will be based on the top-level requests from the scientists, as laid out in the long-term plan (the Science Activity Plan). The detailed trajectory will be provided by the RMOC Flight Dynamics Team as a state vector, i.e. position and velocity relative to the comet's mass center as a function of time.

The 'environment' refers to the resulting conditions like distance to the nucleus, illumination conditions, and other constraints that need to be taken into account.

Operational constraints for Rosetta will be e.g. the pointing constraints as described at the beginning of this paper, the fact that no eclipses are allowed, power and data volume limitations of the spacecraft, and the number of telecommands on the on-board mission timeline which is limited.

\section{TARget List And Science Constraints}

It is currently assumed that during the first phase of the mission, when the comet is characterized, targets are defined on the surface. These targets can be active areas, craters, or other landmarks. They can be given names and will have their positions defined in a cometocentric coordinate system. These targets can then be used to plan scientific observations. With each observation, constraints can be given for each target. At the time being the envisaged constraints are illumination conditions, the distance range to target during the observation, any requests for repeated observations (e.g. once per week), and a priority for the observation.

Other than on previous planetary missions, it is envisaged that pointing requests can be given relative to target names. This will allow to uniquely identify a pointing request without giving absolute numbers (i.e. a pointing direction in some coordinate system). This would not be possible for longer times into the future as the trajectory of the spacecraft cannot be predicted very well into the future, as discussed at the beginning of the paper.

Observations not related to targets will also be defined.

\section{Future Developments}

The process decribed above and the tools which support it were used successfully mainly on Venus Express and partially by Mars Express and Smart-1. Mainly on Smart-1, ESA introduced a more science-driven planning approach which is 
currently considered to be used on other solar system missions including Rosetta. The concept there is to ask the experiment teams to provide scientific observation requests rather than payload commands. An example could be 'Observe the active region at coordinates $\mathrm{a} / \mathrm{b}$ for at least 5 minutes with the sun between 30 and 60 deg elevation, at a distance of less than $5 \mathrm{~km}$. Obtain images in the silicate filter set.'. On Smart-1, this concept was implemented via a web-based interface for the PI teams where they could formulate their science requests and which operational 'blocks' they want to have executed. The operational blocks are pre-defined and stored in a central database. At the SOC, a software tool linked to an environmental simulator would search for so-called target observation windows (TOW) which fulfill the requirements. On Smart-1, the operational blocks were then manually put in place. Currently, studies are ongoing to produce an automated scheduler which could actually optimize the operational plan.

This concept would ensure that the experimenter teams can focus on the science they want to perform, rather than dealing with engineering issues. However, more studies are needed to show the feasibility of implementing such an approach.

\section{Summary}

This paper outlines the constraints and design drivers for the science planning concept for ESA's comet mission Rosetta, with a special emphasis on how the science requirements are mapped to operations. The underlying concept is the 'Science Themes concept' which is already used on other ESA missions, e.g. Venus Express (Titov et al., 2006). It will ensure that even in a highly dynamical environment as around a comet the mission will return good scientific results.

\section{References}

Dhiri, V., Wirth, K., and Koschny, D.: 2006, Proc. SpaceOps Conference 2006, 19-23 Jun 2006, Rome, Italy (accepted).

Glassmeier, K.-H., Boehnhardt, H., Koschny, D., Kührt, E., and Richter, I.: this issue, doi: 10.1007/s11214-006-9140-8.

Titov, D., Svedhem, H., Koschny, D., Hoofs, R., Barabash, S., Bertaux, J.-L., et al.: 2006, Planetary Space Science (accepted).

Wirth, K., Dhiri, V., Koschny, D., Diaz del Rio, J., Zender, J., and Schwehm, G.: 2006, Proc. SpaceOps Conference 2006, 19-23 Jun 2006, Rome, Italy (accepted). 